\title{
Enzyme-coated metal-organic framework shell for synthetically adaptive cell survival
}

\author{
Kang Liang, ${ }^{*}$ Joseph J. Richardson, Christian J. Doonan, Xavier Mulet, Yi Ju, Jiwei Cui, Frank Caruso, \\ and Paolo Falcaro*
}

\begin{abstract}
A bioactive synthetic porous shell was engineered to enable cells to survive in an oligotrophic environment. Eukaryotic cells (yeast) were firstly coated with a $\beta$-galactosidase ( $\beta$-gal), then a metalorganic framework (MOF) film was crystallized on the enzyme coating producing a bioactive porous synthetic shell. The $\beta$-gal was an essential component of the bioactive shell as it generated nutrients (i.e. glucose and galactose) required for cell viability from nutrient deficient media (lactose-based). In addition the porous MOF coating carried out other vital functions, such as: 1) shielding the cells from cytotoxic compounds and radiation, 2) protecting the non-native enzymes ( $\beta$-gal in this instance) from degradation and internalization, 3 ) allowing for the diffusion of molecules essential for the survival of the cells. Indeed, this bioactive porous shell enabled the survival of cells in simulated extreme oligotrophic environments for more than 7 days, leading to a decrease in cell viability less than $30 \%$, versus a $99 \%$ decrease for naked yeast. When returned to optimal growth conditions and the bioactive porous exoskeleton could be removed and the cells regained full growth immediately. Alternative to synthetic biology or genetic modification, the construction of bioactive coatings represents a conceptually new and promising approach for the nextgeneration of cell-based research and application.
\end{abstract}

Living organisms are sensitive to their environment and subtle changes in nutrient level, temperature, pressure, moisture, salinity, and $\mathrm{pH}$ can disrupt their biological functions, thereby leading to cell death. ${ }^{[1-3]}$ To address this challenge, primary

[*] Dr. K. Liang

School of Chemical Engineering, The University of New South Wales, Sydney, NSW 2052, Australia

Graduate School of Biomedical Engineering, The University of New South Wales, Sydney, NSW 2052, Australia

E-mail: kang.liang@unsw.edu.au

Dr. K. Liang, Dr. J. J. Richardson, Dr Xavier Mulet

CSIRO Manufacturing

CSIRO Private Bag 10, Clayton South, Victoria 3169, Australia

Prof. C. J. Doonan

School of Chemistry and Physics

The University of Adelaide

Adelaide, South Australia 5005, Australia

Dr. J. J. Richardson, Dr. Y. Ju, Dr. J. Cui, Prof. F. Caruso

ARC Centre of Excellence in Convergent Bio-Nano Science and

Technology, and the Department of Chemical and Biomolecular

Engineering

The University of Melbourne, Parkville, Victoria 3010, Australia

Dr. J. Cui

Key Laboratory of Colloid and Interface Chemistry of Ministry of

Education, and the School of Chemistry and Chemical Engineering,

Shandong University, Jinan 250100, China.

Prof. P. Falcaro

Institute of Physical and Theoretical Chemistry

Graz University of Technology, Stremayrgasse 9, Graz 8010,

Austria

E-mail: paolo.falcaro@tugraz.at

Supporting information for this article is given via a link at the end of the document. research in genetic engineering and synthetic biology focuses on understanding and engineering genetic codons to enhance cellular adaptive responses to environment changes. ${ }^{[4,5]}$ Generally, synthetic biology aims to develop new circuit design principles that involve multistep procedures including creating and analyzing computational models, constructing genetic circuits, evaluating the performance of the genetically engineered cells, and refining the design process. ${ }^{[6]}$ Although recent developments in precision genome engineering, such as zinc finger proteins (ZFPs), transcription activator-like (Tal) effectors and clustered regularly interspaced short palindromic repeats (CRISPRs), have significantly enhanced the versatility of gene editing, these techniques still face several challenges including a high level of complexity, difficulties in characterization, standardization and modularity, noise, epigenetics, mutations, and a risk of accidental release into the wild, amongst other things. ${ }^{[6]}$ Therefore, developing a generalized, non-genetic engineering alternative to temporarily enhance the adaptability of cells is highly desired. Recently, artificial nanocoatings have appeared as an approach to mimic the robustness of cell membranes and walls found in some extremophiles, while avoiding genetic modification. ${ }^{[7-9]}$ Nanocoating cell surfaces creates a physical barrier between the cell and the external environment while still allowing nutrient exchange. These nanocoatings have improved cellular tolerance against heat, ${ }^{[10,11]}$ UV radiation, ${ }^{[12,13]}$ toxins, ${ }^{[14-17]}$ osmotic pressure, ${ }^{[18,19]}$ and mechanical stress. ${ }^{[20,21]}$ Although nanocoatings have demonstrated protective abilities, their integration with active biomacromolecules is a challenge. This prevents the bioengineering of such coatings with extrinsic bioactive functionalities that could impart artificial adaptive ability thus overcoming original biological limitations. For example, eukaryotic cells lack the ability to harvest energy from nutrient depleted environments, ${ }^{[22]}$ but providing a cells with a bioactive protective nanocoating capable of converting depleted media into usable nutrients could reveal new opportunities in therapy, ${ }^{[23]}$ diagnostics, ${ }^{[24]}$ stress-response, ${ }^{[25,26]}$ biocatalysis, ${ }^{[27]}$ and could also revolutionize the dairy and pharmaceutical industries. ${ }^{[28,29]}$

Among the wide choice of synthetic coatings, metal-organic frameworks (MOFs) are a class of porous materials that offer several unique advantages for engineering eukaryotic cells to survive in inhospitable environments. ${ }^{[30]}$ MOFs can be thermally and chemically robust, ${ }^{[31]}$ and can be synthesized via modular approaches that facilitate the fine-tuning of pore shape, ${ }^{[32]}$ chemical functionality, ${ }^{[33,34]}$ and size. ${ }^{[35]}$ More importantly, MOFs hold promise for biomedical applications, ${ }^{[36]}$ as they can be constructed from bio-friendly building blocks ${ }^{[37]}$ under physiological conditions. ${ }^{[38]}$ Here, we demonstrate for the first time that an active biocomposite MOF shell can be integrated with cells; as a result eukaryotic cells can survive in cytotoxic environments without the need of essential nutrients. The proofof-concept was obtained by coating living eukaryotic cells with a 
protective MOF exoskeleton functionalized with exogenous enzymes that do not exist naturally in the cells. This enzyme is capable of converting disaccharides, which can not be utilized by cells, into monosaccharides which are metabolisable nutrients for the cells. Notably the MOF shell allows for the diffusion of nutrients to the living cells (Scheme 1) while providing a selective barrier that can protect both cells and non-native enzymes. Saccharomyces cerevisiae (baker's yeast) was selected in this study, as these cells do not have a lactose metabolic system, therefore genetic enginering aproaches are currently used to construct lactose-consuming $S$. cerevisiae strains. ${ }^{[39,40]}$ In the proposed study native $S$. cerevisiae coated with $\beta$-galactosidase ( $\beta$-gal) and zeolitic imidazolate framework-8 (ZIF-8) showed cell survival for more than 7 days in an environment lacking an essential nutrient, namely glucose. The $\beta$-gal protected by the
MOF coating ( $\beta$-gal/ZIF-8) was able to continuously produce glucose and galactose from environmental lactose and thereby maintain cell viability, even in conditions that would normally kill the yeast (exposure to the lytic enzyme, lyticase) or destroy the $\beta$-gal (exposure to a protease cocktail). Although ZIF-8 was used in this study, other MOFs could also be employed with careful consideration of the solubility of the MOF ligands and their cytotoxicity. The construction of a biologically active biocomposite MOF coating, that can be easily removed, demonstrates metabolisable a new and promising approach for facile, nongenetic, cellular manipulation.

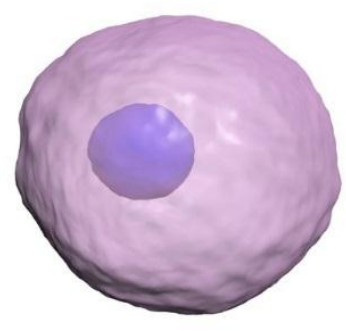

Re-growth

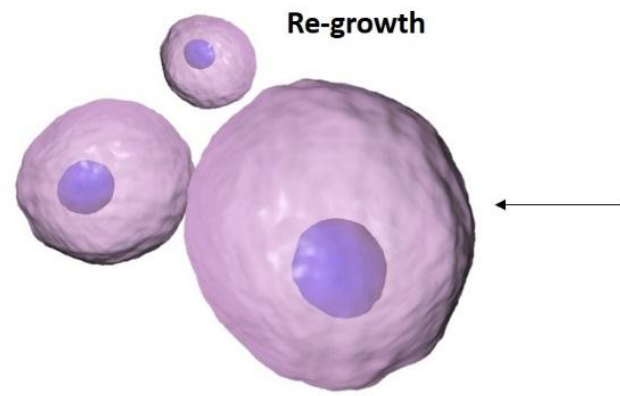

Functional enzyme coating
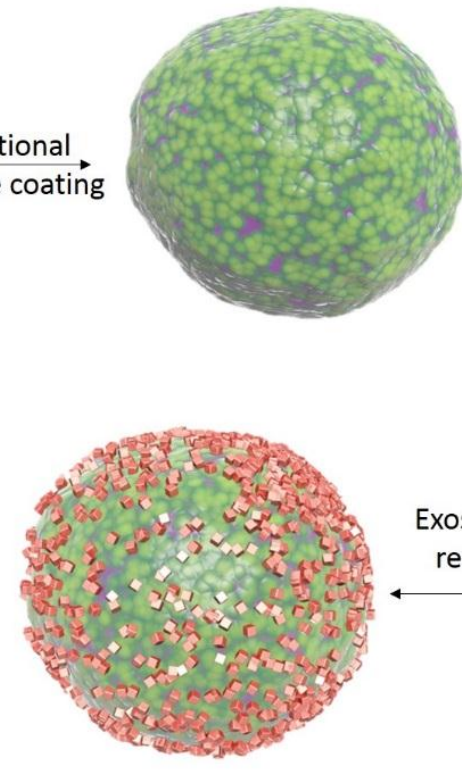

Exoskeleton removal

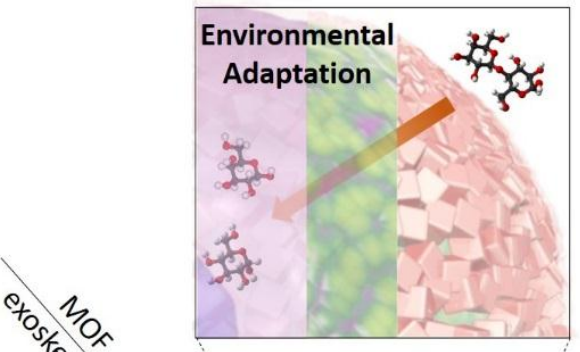

Scheme 1. Schematic illustration showing the construction and removal of the bioactive porous ( $\beta$-gal/ZIF-8) shell for synthetically adaptive cell survival. A living cell is coated with a layer of bioactive exogenous enzymes ( $\beta$-galactosidase, $\beta$-gal) capable of bioprocessing lactose resources to generate essential nutrients for the cells (e.g. glucose). The enzymes and cells are both protected by an artificial exoskeleton composed of porous metal-organic frameworks (MOFs), allowing cells to survive in hostile environments. In the current study the MOF chosen is ZIF-8. Upon removal of the ZIF-8 coating, the cells regain their full growth potential.

Yeast cells were first coated with $\beta$-galactosidase by electrostatically absorbing the cationic enzymes on the anionic cell surface in phosphate buffered saline (PBS). ${ }^{[41,42]}$ Confocal laser scanning microscopy (CLSM) was used to confirm the full surface coverage of the enzymes on the yeast cells (Figure 1a-b and Figure S1, Supporting Information). Although ZIF-8 coatings have previously been grown on naked yeast. ${ }^{[30]}$, the modification of yeast cells with a protein-rich surface could also favor the attraction of MOF precursors including both cations and ligands, leading to the rapid biomimetic crystallization of a MOF shell. ${ }^{[38,43]}$ Here, the biomimetic growth of a MOF shell was achieved by incubating the enzyme-coated cells in an aqueous solution containing 2-methylimidazole and zinc acetate to form ZIF-8. After $10 \mathrm{~min}$, the coated cells were removed from the solution and washed with water to remove the excess MOF precursors. CLSM was employed to visualize the MOF coating in solution after infiltration with the fluorescent dye, Alexa Fluor 647. A continuous fluorescent signal around each individual cells was observed, indicating the formation of a homogeneous MOF shell on individual cells. Cell viability assays using fluorescein diacetate (FDA) as a fluorescent indicator showed a negligible decrease in fluorescent intensity after coating the cells in the $\beta$-gal/ZIF-8 exoskeleton, indicating the enzyme and MOF coating process had a minimal impact on cell viability (Figure 1a-d and Figure S2, Supporting Information). The structure of the coating was assessed under small-angle X-ray scattering (SAXS) and the resulting diffraction pattern yielded peaks analogous both in position and relative intensity to pure ZIF-8 crystals (Figure 1e), thereby confirming the crystalline nature of the $\beta-g a l / Z I F-8$ exoskeleton. The morphology and elemental distribution of the $\beta$ gal/ZIF-8 exoskeleton was investigated using scanning electron microscopy (SEM) and energy-dispersive X-ray spectroscopy 
(EDS). SEM images revealed that the surface of individual yeast cells was covered by a layer of nanocrystals (Figure $1 \mathrm{f}$ and Figure S3, Supporting Information), while elemental mapping confirmed a homogeneous distribution of overlapping $O$ (from the yeast and $\beta$-gal), $C$ (from the yeast, $\beta$-gal and ZIF-8), N (from the yeast, $\beta$ gal and ZIF-8), and Zn (from the ZIF-8) signals (Figure S4, Supporting Information). The SEM/EDS data was consistent with the high-resolution optical cross sections obtained from CLSM, providing further evidence of a homogeneous coating of the $\beta$ $\mathrm{gal} / \mathrm{ZIF}-8$ exoskeleton over each individual cell. The thickness of the exoskeleton measured on different cracked shell was $100 \mathrm{~nm}$ $\pm 10 \mathrm{~nm}$ (as measured from SEM cross-sections Figure S5, Supporting Information), which is consistent with pure ZIF-8 coatings grown directly on yeast cells. ${ }^{[30]}$
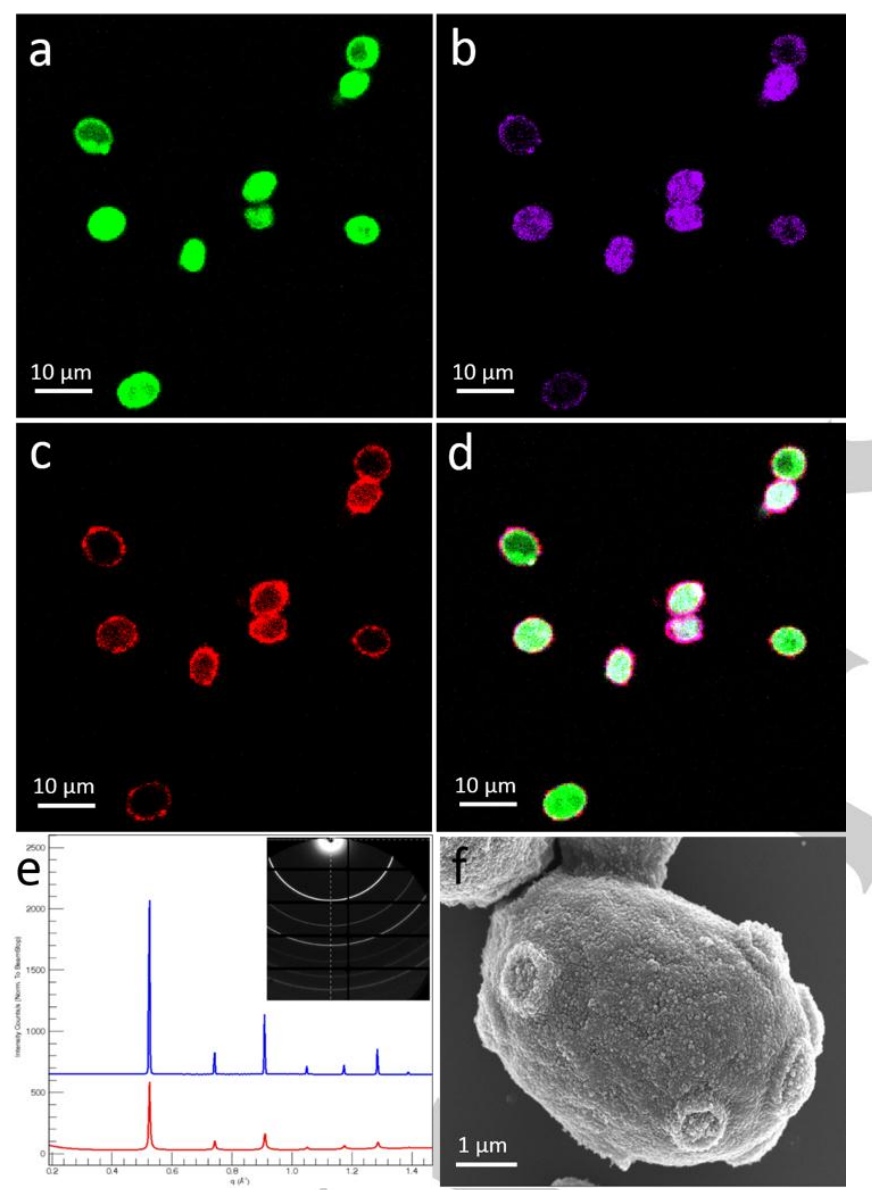

Figure 1. CLSM cross section images of (a) live yeast cells, (b) $\beta$-gal coating, (c) ZIF-8 coating and (d) merged image. The living yeast cells catalyzed FDA into fluorescein (green), $\beta$-galactosidase was labelled with Alexa Fluor 568 (purple), and the ZIF-8 coatings were labelled by infiltration of Alexa Fluor 647 (red), respectively. (e) Synchrotron small-angle X-ray scattering (SAXS) diffraction patterns of standard ZIF-8 crystals (blue) and $\beta$-gal/ZIF-8 coated yeast cells (red). Inset: $2 \mathrm{D}$ SAXS pattern of the $\beta$-gal/ZIF- 8 coated yeast cells (measured using a $0.5 \mathrm{~mm}$ capillary as a sample holder). (f) SEM image of a yeast cell coated with the $\beta$-gal/ZIF-8 exoskeleton.

Saccharomyces cerevisiae must consume specific monoand disaccharides for their metabolic needs, ${ }^{[44]}$ and we hypothesized that the introduction of new non-native enzymes on a synthetic porous shell could allow yeast cells to survive in oligotrophic environments that are otherwise inhospitable. We first determined whether the new coatings could produce essential nutrients for yeast survival. $\beta$-gal is a glycoside hydrolase enzyme that converts lactose into glucose and galactose, both of which can be consumed by yeast as essential nutrients. ${ }^{[45]}$ Yeast cells coated with $\beta$-gal/ZIF-8 were incubated in modified cell media containing lactose instead of glucose to simulate a nutrient deficient environment. The production of glucose and galactose was then monitored by an external enzyme cascade reaction using an enzyme cocktail composed of glucose oxidase, horseradish peroxidase, and a colorimetric indicator, pyrogallol (Figure S6, Supporting Information). ${ }^{[46]}$ Accordingly, absorbance at $420 \mathrm{~nm}$ was monitored as it is the result of the catalytic conversion of pyrogallol. The continuous increase in intensity (Figure S7, Supporting Information) demonstrated that 1) lactose was able to diffuse through the ZIF-8 coating; 2) the MOF coated $\beta$-gal was acting as a biocatalyst for the production of essential sugars for the yeast cells; 3 ) the biocatalyzed glucose diffused through the ZIF-8 shell. In contrast, the conversion of lactose was not observed in the absence of either the $\beta$-gal, as no glucose was produced, or the ZIF-8 coating, as the yeast likely internalized and degraded $\beta$-gal (Figure S7, Supporting Information). This data highlights the importance of the synergistic effect achieved by engineering a MOF shell with an active enzyme. To further ascertain the importance of the current composition and structuralization of the coating pure ZIF- 8 crystals and free $\beta$ gal@ZIF-8 particles were exposes to lactose, however glucose conversion was not detected (Figure S8, Supporting Information). The inability of $\beta$-gal@ZIF-8 to process lactose is consistent with previous findings of molecular diffusion in ZIF-8. ${ }^{[47]}$ Additionally this suggests that the diffusion of lactose through the ZIF-8 cell coatings is likely due to the inter-particle spacing rather than through the pore network. Based on the degradation of free $\beta$-gal from yeast, we suspect that the ZIF-8 coatings immobilize the enzymes on the cell surface, thereby preventing the internalization and digestion of the enzymes by the yeast cells. This confirms the importance of the $\beta$-gal@ZIF-8 structure for bioactivity.

Having demonstrated the potential of the bioactive porous coating in generating essential nutrients, we were motivated to investigate the potential of these cells in surviving extreme and nutrient deficient environments. To simulate extreme environments, toxic compounds that are detrimental to yeast cells and/or $\beta$-gal, including a lytic enzyme, lyticase, ${ }^{[48]}$ or a proteolytic enzyme cocktail, protease, ${ }^{[49]}$ were introduced to glucose deficient cell media containing lactose. Both naked and coated yeast cells were cultured in the modified cell media, and the cell viability was monitored using FDA as a fluorescent live cell indicator over time. Using a combination of high-throughput flow cytometry and high resolution optical microscopy, ${ }^{[50]}$ the living cells could easily be separated from the dead cells (Figure S9, Supporting Information). The viability of each sample was monitored for up to 7 days, and the percentage of living cells at each time point was calculated and plotted in Figure 2. Without any toxic enzymes in the media, the viability of the naked cells rapidly decreased within days (Figure S10, Supporting Information), suggesting that the naked cells could not survive during prolonged culturing with only lactose 
as a nutrient source. The immediate death of naked cells was more apparent when lyticase was present in the media, as lyticase can lyse yeast cells in a manner of hours. ${ }^{[30]}$ The biocomposite MOF coated yeast cells only showed a small drop $(<30 \%)$ in viability even after 7 days in the modified cell media with or without lyticase (Figure 2 and Figure S11, Supporting Information), suggesting that the engineered exoskeletons are effective in generating essential nutrients for prolonged cell survival while also protecting the yeast from toxic enzymes. To further ascertain the stability and robustness of the coatings and yeast against toxic enzymes in nutrient deficient media, we studied the survival of these cells in the presence of proteases. Proteases are a class of enzymes that catalyze the breakdown of proteins through the hydrolysis of peptide bonds, and if uncontrolled, can destroy the proteinaceous components of cells and tissues. ${ }^{[49]}$ Moreover, protease is capable of rapidly degrading enzymes like $\beta$-gal in solution. When protease was introduced into the cell culture, the viability of naked cells decreased to $10 \%$ after one day. In contrast, more than $70 \%$ of the $\beta$-gal/ZIF-8 coated cells remained viable even after 7 days (Figure 2 and Figure S12, Supporting Information). This result clearly indicates that the ZIF-8 coating was able to protect both the $\beta$-gal and the living cells from proteolytic attack. The c.a. $30 \%$ drop in viability of the biocomposite MOF coated yeast cells at day 7 is likely due to the extensive consumption of lactose in the cell media, leading to limited glucose generation. Enzyme cascade assay results indicated that almost $90 \%$ of lactose has been consumed by the $\beta$-gal/ZIF-8 coated yeast cells by day 7 (Figure S13, Supporting Information). By day 10 , a majority ( 70\%) of the $\beta$-gal/ZIF-8 coated yeast cells had lost their viability due to the complete depletion of lactose. The biocomposite MOF coated yeast cells were exposed to oligotrophic media containing both lyticase and protease for $48 \mathrm{~h}$, and finally exposed to UV-C radiation, as DNA and proteins in living organisms can be damaged by UV-C. ${ }^{[1]} \beta$ gal/ZIF-8 coated yeast cells showed an $89 \%$ viability versus $5 \%$ viability for the naked yeast after the multiple environmental exposures (Figure S14, Supporting Information). The protection against UV can be attributed to absorbance of ZIFs in the UV region. ${ }^{[52]}$ Therefore our results demonstrate that the biocomposite MOF coated yeast cells can survive for an extended period of time in nutrient depleted and adverse environments. In addition, the metabolic activity of the $\beta$-gal/ZIF- 8 coated yeast cells in nutrient depleted and toxic environments was studied by measuring the $\mathrm{CO}_{2}$ production rate as a result of anaerobic respiration (Figure S15, Supporting Information).[53] Results indicated that the biocomposite MOF coated yeast cells were able to produce $\mathrm{CO}_{2}$ at a similar rate to those naked cells in glucose. In contrast, naked yeast cells in lactose did not show obvious $\mathrm{CO}_{2}$ production without the functional coatings.

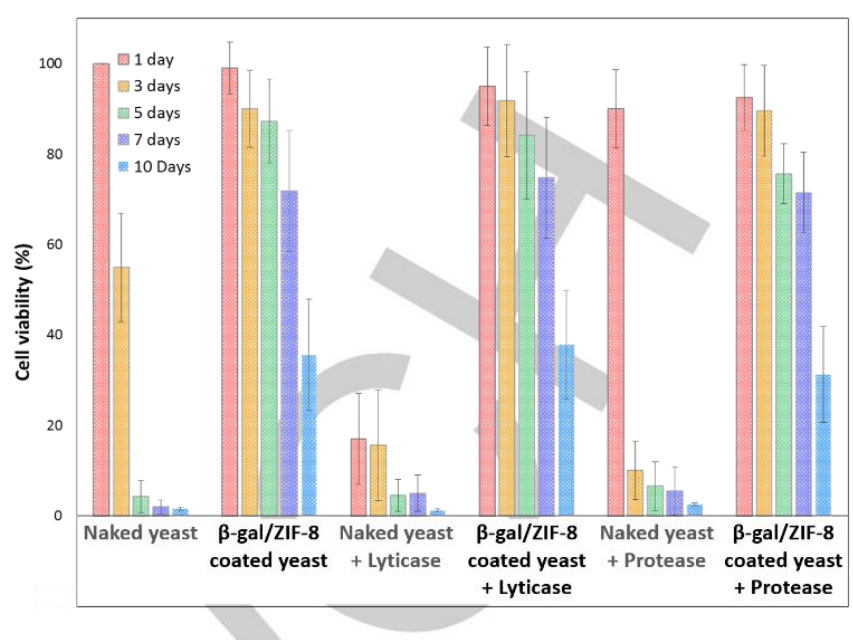

Figure 2. Relative cell viability (\%) of naked yeast and biocomposite MOF ( $\beta$ gal/ZIF-8) coated yeast cells in oligotrophic cell media containing lactose. Cytotoxic enzymes such as lyticase or protease were added to simulate inhospitable environments. Cell viability was normalized against the viability of naked yeast at day one.

To gain further understanding of the impact of the exoskeleton on the coated cells after living in stressed environments for prolonged periods, the MOF coating was removed by the addition of EDTA following 7 days of culturing in lactose media, and the released cells were transferred to standard culture media containing glucose. Cell proliferation was monitored by optical density measurements at $600 \mathrm{~nm}\left(\mathrm{OD}_{600}\right) \cdot{ }^{[54]}$ Compared with the naked yeasts, the released cells skipped the lag phase and immediately regained full proliferation potential (growth phase), similar to our previous study. ${ }^{[30]}$ In addition, there is no obvious reduction of the growth rate compared to naked yeast cells at the growth phase stage (Figure 3). These results clearly demonstrated that the large majority of yeast cells coated with the active biocomposite MOF shell remained healthy after surviving a week in simulated extreme, nutrient-deficient environment. Given that the MOF coatings can be easily removed on demand, ${ }^{\left[{ }^{[0]}\right.}$ this coating strategy shows new potential for engineering selective adaptability in living cells, without the contamination risks associated with genetic engineering. 


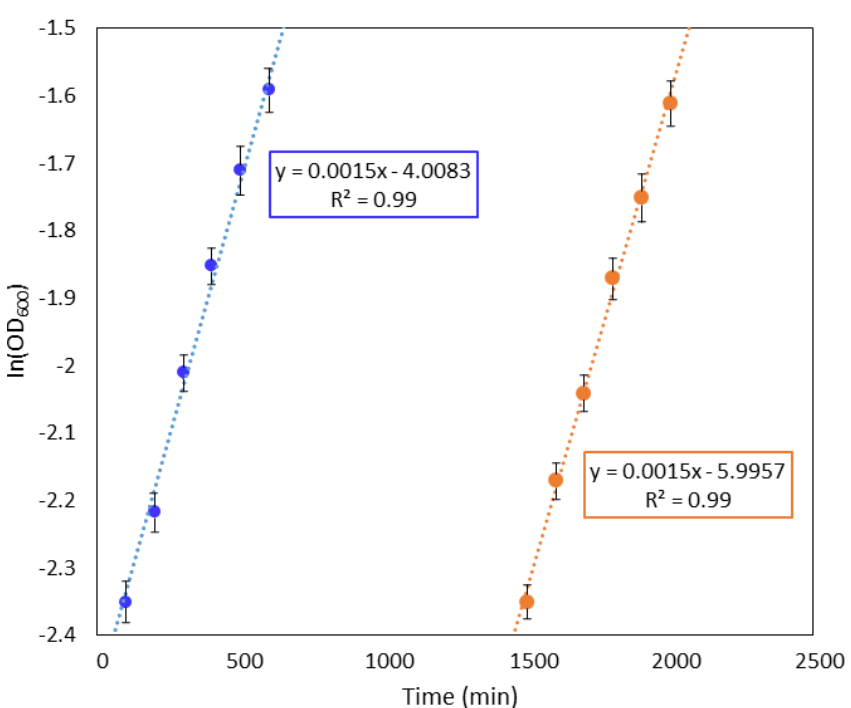

Figure 3. Growth phase linear-fitted plot of $\ln \left(\mathrm{OD}_{600}\right)$ vs. time for $\beta$-gal/ZIF-8 coated yeast cells after ZIF-8 removal by EDTA (blue) and naked yeast as a control (orange). The growth rate is calculated from the slope of the linear fitted line. After the bioactive porous coating removal the yeast fully retained their original growth rate.

In conclusion, we have demonstrated for the first time that exogenous enzymes and MOFs can be synergistically engineered directly on living systems. The resulting bioactive shell allows cells to survive in food deficient environments in presence of other adverse conditions (toxic agents and UV irradiation). Proof-of-concept experiments show that the enzyme/MOF coating was able to generate essential nutrients for the cells, while the MOF protected both the cells and non-native enzymes from harmful compounds and still allowed for the transport of nutrients. In addition, the cells regain their full growth potential following the removal of the biocomposite MOF exoskeleton. Based on our previous reports, which demonstrated that different cells (e.g., prokaryotic and eukaryotic) can be coated with MOFs, we anticipate that different cells, enzymes, and MOFs could be employed to expand the scope and application of adaptive coatings for cell survival. The construction of hybrid and synthetically adaptive coatings for living represents a potentially novel approach for addressing challenges in biology and biotechnology.

\section{Experimental Section}

Experimental Details including materials, coating formation on cell surfaces, enzyme cascade assay, cell viability test, cell proliferation assay, and instrumentation are documented in the Supporting Information.

\section{Acknowledgements}

J.J.R. acknowledge CSIRO OCE Science Scheme. This research was conducted and funded by the Australian Research Council (ARC) Centre of Excellence in Convergent Bio-Nano Science and
Technology (Project Number CE140100036) and funded by the ARC under the Australian Laureate Fellowship (FL120100030) scheme. This work was performed in part at the Materials Characterisation and Fabrication Platform (MCFP) at the University of Melbourne and the Victorian Node of the Australian National Fabrication Facility (ANFF). Part of this research was conducted at the SAXS/WAXS beamline at the Australian Synchrotron.

Keywords: biomimetics $\cdot$ synthetic cells $\cdot$ cytoprotection $\cdot$ ZIF-8 - hybrid cells

[1] D. B. Roszak, R. R. Colwell, Microbiol. Rev. 1987, 51, 365-79.

[2] J. Liu, J. Wen, Z. Zhang, H. Liu, Y. Sun, B. Lowell, B. Spiegelman, D. Patel, K. Franklin, H. Jiang, et al., Microsystems Nanoeng. 2015, $1,15020$.

[3] M. Ayrapetyan, T. C. Williams, J. D. Oliver, E. P. Abraham, E. Chain, R. R. Colwell, J. T. Lennon, S. E. Jones, D. Pinto, et al., et al., Trends Microbiol. 2015, 23, 7-13.

[4] D. Endy, Nature 2005, 438, 449-453.

[5] A. K. Kim, R. DeRose, T. Ueno, B. Lin, T. Komatsu, H. Nakamura, T. Inoue, Sci. Signal. 2016, 9.

[6] P. E. M. Purnick, R. Weiss, Nat. Rev. Mol. Cell Biol. 2009, 10, 41022.

[7] Z. Liu, X. Xu, R. Tang, Adv. Funct. Mater. 2016, 26, 1862-1880

[8] J. H. Park, S. H. Yang, J. Lee, E. H. Ko, D. Hong, I. S. Choi, Adv. Mater. 2014, 26, 2001-2010.

[9] I. Drachuk, O. Shchepelina, M. Lisunova, S. Harbaugh, N. KelleyLoughnane, M. Stone, V. V. Tsukruk, ACS Nano 2012, 6, 42664278 .

[10] G. Wang, L. Wang, P. Liu, Y. Yan, X. Xu, R. Tang, ChemBioChem 2010, 11, 2368-2373.

[11] E. H. Ko, Y. Yoon, J. H. Park, S. H. Yang, D. Hong, K.-B. Lee, H. K Shon, T. G. Lee, I. S. Choi, Angew. Chem. Int. Ed. Engl. 2013, 52, 12279-82.

[12] B. Wang, P. Liu, Y. Tang, H. Pan, X. Xu, R. Tang, PLoS One 2010, 5, e9963.

[13] J. H. Park, K. Kim, J. Lee, J. Y. Choi, D. Hong, S. H. Yang, F. Caruso, Y. Lee, I. S. Choi, Angew. Chem. Int. Ed. Engl. 2014, 53, 12420-5.

[14] B. Wang, P. Liu, W. Jiang, H. Pan, X. Xu, R. Tang, Angew. Chemie Int. Ed. 2008, 47, 3560-3564.

[15] D. Hong, H. Lee, E. H. Ko, J. Lee, H. Cho, M. Park, S. H. Yang, I. S. Choi, M. T. Stephan, J. J. Moon, et al., Chem. Sci. 2015, 6, 203208.

[16] J. Lee, J. Choi, J. H. Park, M.-H. Kim, D. Hong, H. Cho, S. H. Yang, I. S. Choi, Angew. Chem. Int. Ed. Engl. 2014, 53, 8056-9.

[17] H.-K. Na, M.-H. Kim, J. Lee, Y.-K. Kim, H. Jang, K. E. Lee, H. Park, W. Do Heo, H. Jeon, I. S. Choi, et al., Nanoscale 2013, 5, 1669.

[18] S. H. Yang, K.-B. Lee, B. Kong, J.-H. Kim, H.-S. Kim, I. Choi, Angew. Chemie Int. Ed. 2009, 48, 9160-9163.

[19] R. Kempaiah, S. Salgado, W. L. Chung, V. Maheshwari, Chem. Commun. (Camb). 2011, 47, 11480-2.

[20] A. Matsuzawa, M. Matsusaki, M. Akashi, Langmuir 2013, 29, 73627368. 
C. Ye, Z. A. Combs, R. Calabrese, H. Dai, D. L. Kaplan, V. V.

Tsukruk, Small 2014, 10, n/a-n/a.

[22] R. Dixon, D. Kahn, Nat. Rev. Microbiol. 2004, 2, 621-631.

[23] D. W. Green, G. Li, B. Milthorpe, B. Ben-Nissan, Mater. Today 2012, 15, 60-66.

[24] J. A. Irwin, Environ. Technol. 2010, 31, 857-869.

[25] M. Dassanayake, D.-H. Oh, J. S. Haas, A. Hernandez, H. Hong, S. Ali, D.-J. Yun, R. A. Bressan, J.-K. Zhu, H. J. Bohnert, et al., Nat. Genet. 2011, 43, 913.

[26] G. Feller, C. Gerday, Nat. Rev. Microbiol. Publ. online 01 December 2003; / doi10.1038/nrmicro773 2003, 1, 200.

[27] S. G. Burton, D. A. Cowan, J. M. Woodley, Nat. Biotechnol. Publ. online 01 January 2002; / doi10.1038/nbt0102-37 2002, 20, 37.

[28] E. Pennisi, Science 1997, 276, 705-6.

[29] B. van den Burg, Curr. Opin. Microbiol. 2003, 6, 213-218.

[30] K. Liang, J. J. Richardson, J. Cui, F. Caruso, C. J. Doonan, P. Falcaro, Adv. Mater. 2016, 28, 7910-7914.

[31] K. S. Park, Z. Ni, A. P. Côté, J. Y. Choi, R. Huang, F. J. UribeRomo, H. K. Chae, M. O'Keeffe, O. M. Yaghi, Proc. Natl. Acad. Sci. U. S. A. 2006, 103, 10186-91.

[32] E. D. Bloch, W. L. Queen, R. Krishna, J. M. Zadrozny, C. M. Brown, J. R. Long, Science 2012, 335, 1606-10.

[33] H. Deng, C. J. Doonan, H. Furukawa, R. B. Ferreira, J. Towne, C. B. Knobler, B. Wang, O. M. Yaghi, Science 2010, 327, 846-50.

[34] K. Ariga, J. Li, J. Fei, Q. Ji, J. P. Hill, Adv. Mater. 2016, 28, 12511286.

[35] H. Furukawa, K. E. Cordova, M. O’Keeffe, O. M. Yaghi, Science 2013, 341, 1230444.

[36]

P. Horcajada, R. Gref, T. Baati, P. K. Allan, G. Maurin, P. Couvreur, G. Férey, R. E. Morris, C. Serre, Chem. Rev. 2012, 112, 1232-68. R. A. Smaldone, R. S. Forgan, H. Furukawa, J. J. Gassensmith, A. M. Z. Slawin, O. M. Yaghi, J. F. Stoddart, Angew. Chem. Int. Ed. Engl. 2010, 49, 8630-4.

[38] K. Liang, R. Ricco, C. M. Doherty, M. J. Styles, S. Bell, N. Kirby, S. Mudie, D. Haylock, A. J. Hill, C. J. Doonan, et al., Nat. Commun.
2015, 6, 7240 .

[39] M. Rubio-Texeira, M. Arévalo-Rodríguez, J. Luis Lequerica, J. Polaina, J. Biotechnol. 2000, 84, 97-106.

[40] L. Domingues, P. M. R. Guimarães, C. Oliveira, Bioeng. Bugs 2010 1, 164-171.

[41] P. N. Lipke, R. Ovalle, J. Bacteriol. 1998, 180, 3735-3740.

[42] T. Sirec, A. Strazzulli, R. Isticato, M. De Felice, M. Moracci, E. Ricca, C. Wu, A. Mulchandani, W. Chen, S. Lee, et al., Microb. Cell Fact. 2012, 11, 100.

[43] K. Liang, C. Carbonell, M. J. Styles, R. Ricco, J. Cui, J. J. Richardson, D. Maspoch, F. Caruso, P. Falcaro, Adv. Mater. 2015, 27, 7293-8.

[44] R. Lagunas, FEMS Microbiol. Lett. 1993, 104, 229-242.

[45] J. Nielsen, S. Ostergaard, L. Olsson, M. Johnston, Nat. Biotechnol. 2000, 18, 1283-1286.

[46] S. Fornera, K. Yazawa, P. Walde, Anal. Bioanal. Chem. 2011, 401, 2307-2310.

[47] K. Zhang, R. P. Lively, C. Zhang, R. R. Chance, W. J. Koros, D. S. Sholl, S. Nair, J. Phys. Chem. Lett. 2013, 4, 3618-3622.

[48] R. M. Taskova, H. Zorn, U. Krings, H. Bouws, R. G. Berger, Zeitschrift für Naturforsch. C 2006, 61, 347-350.

[49] H. Neurath, Science 1984, 224, 350-7.

[50] K. Liang, S. T. Gunawan, J. J. Richardson, G. K. Such, J. Cui, F. Caruso, Adv. Healthc. Mater. 2014, 3, 1551-1554.

[51] T. A. Slieman, W. L. Nicholson, Appl. Environ. Microbiol. 2001, 67, 1274-9.

[52] G. Kaur, R. K. Rai, D. Tyagi, X. Yao, P.-Z. Li, X.-C. Yang, Y. Zhao, Q. Xu, S. K. Singh, J. Mater. Chem. A 2016, 4, 14932-14938.

[53] J. van den Brink, A. B. Canelas, W. M. van Gulik, J. T. Pronk, J. J. Heijnen, J. H. de Winde, P. Daran-Lapujade, Appl. Environ. Microbiol. 2008, 74, 5710-23.

[54] A. L. Koch, Biochim. Biophys. Acta 1961, 51, 429-441. 


\section{Entry for the Table of Contents}

Layout 1:

\section{COMMUNICATION}

Saccharomyces cerevisiae cells were coated with a bioactive nanoporous shell based on $\beta$-galactosidase and metal-organic framework (MOF). This bioactive shell enables the biocatalysis of glucose from environmental lactose, showing unprecedented cell survival in simulated extreme, nutrient depleted environments.

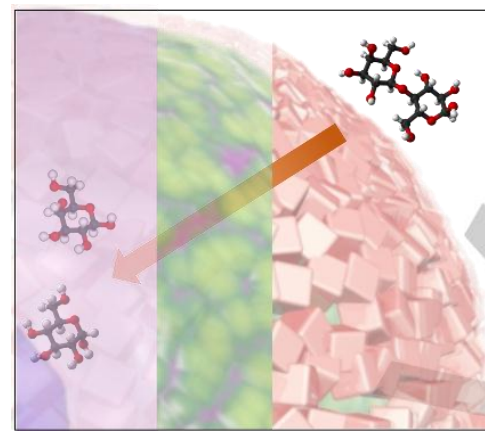

Kang Liang, * Joseph J. Richardson, Christian J. Doonan, Yi Ju, Jiwei Cui, Frank Caruso, and Paolo Falcaro*

Page No. - Page No.

Enzyme-coated metal-organic framework shell for synthetically adaptive cell survival 